\title{
OCORRÊNCIA DE Amblyomma fuscum NEUMANN, 1899 E Amblyomma humerale KOCH, 1844 (ACARI: IXODIDAE) EM Bufo arenalis NO ESTADO DE SÃO PAULO, BRASIL
}

\author{
OCCURENCE OF Amblyomma fuscum NEUMANN, 1899 AND Amblyomma humerale KOCH, 1844 \\ (ACARI: IXODIDAE) IN Bufo arenalis IN THE STATE OF SÃO PAULO, BRAZIL
}

\author{
Afonso Lodovico Sinkoc ${ }^{1}$ João Guilherme Werner Brum²
}

\section{- NOTA -}

\section{RESUMO}

\begin{abstract}
O objetivo deste trabalho é relatar a ocorrência do parasitismo monoespecífico de A. fuscum NEUMANN, 1899 e A. humerale KOCH, 1844 em sapos (Bufo arenalis) no Município de Rosana, Estado de São Paulo, Brasil. Este relato caracteriza um novo hospedeiro e uma nova localização geográfica para estas duas espécies de carrapatos.
\end{abstract}

Palavras-chave: Amblyomma fuscum, Amblyomma humerale, Bufo arenalis, ixodidae, anfíbios, sapos, carrapatos.

\section{SUMMARY}

The objective of this work is to describe the occurence of the monoespecific parasitism of A. fuscum NEUMANN, 1899 and A. humerale KOCH, 1844 in toads (Bufo arenalis) from the County of Rosana, State of São Paulo, Brazil. This is the description of a new host and new geographic site for those two species.

Key words: Amblyomma fuscum, Amblyomma humerale, Bufo arenalis, Ixodidae, amphibians, toads, ticks.

Na família Ixodidae, o gênero Amblyomma possui mais de cem espécies descritas no mundo parasitando um grande número de hospedeiros, das quais, poucas são relatadas como parasitos de animais de sangue frio, sendo estes relatos bastante raros, com escassas citações do parasitismo de anfíbios por carrapatos.

ROBINSON (1926) informou que o $\boldsymbol{A}$. humerale apresentava como distribuição geográfica o Brasil, nos Estados da Bahia, Pará e Norte de Goiás, tendo como hospedeiros tartarugas e tartaruga amazônica (Podocnemis sp.); do A. fuscum afirmou ser conhecida a ocorrência em jibóia (Boa constrictor) na América do Sul. O autor citou ainda Bufo marinus como hospedeiro de A. cajennense, A. dissimile e $A$. goeldii; e Bufo sp. como hospedeiro de A. goeldii e A. rotundatum.

ARAGÃO (1936) informou a ocorrência de A. fuscum sobre Boa constrictor e sobre um lacertídeo, nos Estados de Santa Catarina e Rio Grande do Sul, afirmando ainda que o $A$. humerale ocorre sempre sobre jabuti (Testudo tabulata) nos Estados do Espirito Santo, Pará, Amazonas e Acre, sendo encontrada sempre uma predominância de indivíduos machos sobre fêmeas; informou ainda que $\boldsymbol{A}$. goeldii e $\boldsymbol{A}$. rotundatum são parasitos de animais de sangue frio.

${ }^{1}$ Médico Veterinário, Pós-graduando em Ciências Veterinárias, Faculdade de Veterinária, Universidade Federal do Rio Grande do Sul.

${ }^{2}$ Médico Veterinário, MSc, PhD, Professor Titular, Departamento de Microbiologia e Parasitologia, Instituto de Biologia, Universidade Federal de Pelotas, Caixa Postal 354, 96010-900, Pelotas, RS, Brasil. Autor para correspondência. 
No Rio Grande do Sul, CORRÊA (1954/ 55) informou a ocorrência de A. fuscum sobre cachorro doméstico e tatú, enquanto FREIRE (1972) notificou a ocorrência de $\boldsymbol{A}$. humerale sobre cachorro doméstico proveniente do Município de Jaguarão.

Os carrapatos foram coletados de dois sapos (Bufo arenalis) na margem esquerda do Rio Paranapanema, a aproximadamente cinco quilômetros abaixo da cidade de Rosana, Estado de São Paulo, nos meses de novembro de 1994 e janeiro de 1995.

A identificação específica foi feita utilizando-se a chave proposta por ARAGÃO \& FONSECA (1961), sendo identificados nos dois lotes 13 fêmeas de A. humerale KOCH, 1844 e, 2 machos e 5 fêmeas de $\boldsymbol{A}$. fuscum NEUMANN, 1899, respectivamente em infestações monoespecíficas.

A ocorrência de A. humerale e A. fuscum em anfíbios é fato inédito, uma vez que a primeira espécie já foi citada em quelônios (ROBINSON, 1926; ARAGÃO, 1936) e cachorro doméstico (FREIRE, 1972) e a segunda em cobras (ROBINSON, 1926; ARAGÃO, 1936), lacertídeo (ARAGÃO, 1936), cachorro e tatú (CORRÊA, 1954/55).

$\mathrm{O}$ relato do parasitismo exclusivo por fêmeas de $\boldsymbol{A}$. humerale contraria as informações de ARAGÃO (1936), que afirma ocorrer a predominância de indivíduos machos sobre fêmeas.

A ocorrência de A. fuscum e A. humerale no Estado de São Paulo caracterizaram uma nova localização geográfica para estas espécies, uma vez que a primeira já foi citada na América do Sul
(ROBINSON, 1926) e, no Brasil, nos Estados do Rio Grande do Sul (ARAGÃO,1936; CORRÊA, 1954/55) e Santa Catarina (ARAGÃO, 1936) e a segunda, nos Estados do Rio Grande do Sul (FREIRE, 1972), Bahia, Norte de Goiás (ROBINSON, 1926), Pará (ROBINSON, 1926; ARAGÃO, 1936) Espirito Santo, Amazonas e Acre (ARAGÃO, 1936).

\section{AGRADECIMENTOS}

Agradecemos ao Biólogo Sérgio Rangel Pinheiro do Parque Zoológico Municipal "Quinzinho de Baros", em Sorocaba, SP, pela identificação específica dos hospedeiros.

\section{REFERÊNCIAS BIBLIOGRÁFICAS}

ARAGÃO, H. de B., Ixodidas brasileiros e de alguns paizes limitrophes. Memórias do Instituto Oswaldo Cruz, Rio de Janeiro, v. 31, n. 4, p. 759-844, 1936.

ARAGÃO, H. de B., FONSECA, F., Notas de Ixodologia. VIII lista e chave para os representantes da fauna Ixodológica Brasileira. Memórias do Instituto Oswaldo Cruz, Rio de Janeiro, v. 59, n. 2, p. 115-130, 1961.

CORRÊA, O. Carrapatos determinados no Rio Grande do Sul Biologia, Patologia e Contrôle. Arquivos do Instituto de Pesquisa Veterinárias Desidério Finamor, Porto Alegre, v. 1, p. $35-50,1954 / 55$

FREIRE, J. J. Revisão das espécies da família Ixodidae. Revista de Medicina Veterinária, São Paulo, v. 8, n. 1, p. 1-16, 1972.

ROBINSON, L. E. TICKS. A Monograph of the Ixodoidea. Part IV. The Genus Amblyomma. London: Cambridge University, 1926. 302 p. 